Relations industrielles

Industrial Relations

\title{
Hélène David (sous la direction de), Le vieillissement au travail, une question de jugement!
}

\section{Gilles Guérin}

Volume 46, numéro 3, 1991

URI : https://id.erudit.org/iderudit/050708ar

DOI : https://doi.org/10.7202/050708ar

Aller au sommaire du numéro

Éditeur(s)

Département des relations industrielles de l'Université Laval

ISSN

0034-379X (imprimé)

1703-8138 (numérique)

Découvrir la revue

Citer ce compte rendu

Guérin, G. (1991). Compte rendu de [Hélène David (sous la direction de), Le vieillissement au travail, une question de jugement!]. Relations industrielles / Industrial Relations, 46(3), 671-673. https://doi.org/10.7202/050708ar

Tous droits réservés @ C Département des relations industrielles de l'Université Laval, 1991
Ce document est protégé par la loi sur le droit d'auteur. L’utilisation des services d'Érudit (y compris la reproduction) est assujettie à sa politique d'utilisation que vous pouvez consulter en ligne.

https://apropos.erudit.org/fr/usagers/politique-dutilisation/ 
Despite some shortcomings and rather inconclusive nature of the findings, the book Strikers and Subsidies is a significant work. Hutchens, Lipsky and Stern have integrated institutional information and theoretical insights to develop empirically testable hypotheses pertaining to an important policy issue. Furthermore, even though the authors have tested the hypotheses related to welfare and strikes in specific reference to the United States, the issue is of significant interest in a comparative and international context as well. The authors should be congratulated for opening up a new line of inquiry. And this book should be of great interest and importance to economists, industrial relations specialists, and policy makers concerned with the issue of welfare and industrial conflict.

Basu SHARMA

University of New Brunswick

Le vieillissement au travail, une question de jugement!, sous la direction de Hélène David, Montréal, IRAT, 1990, 209 p., ISBN 2-89248-020-5

En mars 1989, l'Institut de recherche appliquée sur le travail (IRAT) organisait un colloque sur le vieillissement au travail. Les communications des différents intervenants étaient ensuite mises en forme et publiées sous la direction de Hélène David (bulletin $n^{\circ}$ 31-32 de l'IRAT).

Pour qui s'intéresse au vieillissement au travail, ces actes sont extrêmement utiles puisqu'ils rassemblent en un seul document une foule de données et d'informations sur des aspects variés du vieillissement au travail qui n'ont jusqu'ici fait l'objet, au Québec, que de trop rares publications. Certains textes sont supérieurs aux autres mais dans l'ensemble la qualité du matériel présenté est élevée. De nombreux Européens, dont Anne-Marie Guillemard qui a participé à la soirée d'ouverture, ont d'ailleurs contribué au succès de ce colloque. Un seul reproche, ces textes nous apparaissent mal classés avec des titres de section qui ne correspondent pas toujours au contenu des articles correspondants. Ainsi, les articles traitant des politiques publiques et ceux se concentrant sur les politiques organisationnelles auraient gagné à être séparés.

Nous retrouvons dans ces actes les différentes dimensions sous lesquelles le vieillissement au travail peut être étudié. Un premier thème qui ressort, si l'on accepte de bouleverser l'ordre de présentation, est celui de l'analyse du marché du travail des personnes vieillissantes. À partir de statistiques françaises, Serge Volkoff brosse un portrait original de la place qui est faite aux salariés français dans l'appareil de production. Nous aurions souhaité une description du même genre à partir des données de Statistique Canada (par exemple celles rassemblées par Akyeampong ${ }^{1}$ ). Malheureusement, les quelques statistiques canadiennes qui sont rapportées ici et là par certains auteurs ne sont pas analysées en profondeur. Par contre, Lyne McDonald montre comment la situation des travailleurs âgés est fort différente selon qu'ils se trouvent au centre ou à la périphérie (perspective duale) du marché du travail.

Les politiques publiques à l'égard des travailleurs âgés font l'objet d'un traitement abondant. Au Canada, l'assistance-vieillesse, les régimes de retraite publics et les régimes de retraite privés sont présentés par John Myles et Les Teichroew puis par Alain Pilon et Hélène David

1 E. Akyeampong, «Older Workers in the Canadian Labour Market», La population active, novembre 1987, p. 85-120. 
dans des articles très bien documentés mais qui se recoupent partiellement. Martin Kohli et Sven Olsson esquissent le même genre d'analyse dans le cadre spécifique de l'Alilemagne et de la Suède, alors que Bernard Casey reprend son analyse de la retraite anticipée et des transferts de coûts entre les régimes privés et publics, déjà largement publicisée suite à sa publication en 1987 dans la Revue internationale de sécurité sociale ${ }^{2}$.

L'aspect le mieux documenté est sans doute l'analyse de l'impact des conditions de travail sur le vieillissement. De nombreux chercheurs tant français (France Lert, Francis Derriennic) que québécois (Raymond Hétu, Diane Berthelette, Michel Vézina, Alain Vinet et Chantal Brisson) et même finlandais (Juhani Ilmarinen) abordent ce thème, le plus souvent par le biais d'études de cas. Antoine Laville, directeur du laboratoire d'ergonomie physiologique et cognitive à l'École pratique des hautes études (France) fait en quelque sorte la synthèse de ces travaux, lorsqu'il conclut que des conditions de travail sévères, associées à un travail physique lourd, à faible contenu, provoquent un vieillissement accéléré des fonctions physiologiques alors qu'à l'inverse un contenu de travail très diversifié et des conditions d'exécution modérées n'accentuent pas le vieillissement biologique habituel et développent la compétence.

La gestion du vieillissement, quoique l'expression n'est pas utilisée comme telle, est abordée avec l'excellent article de Catherine Cailloux-Teiger sur la formation et un article très global de John Mathews qui décrit tout autant les politiques publiques australiennes que les nouvelles perspectives (notamment celle de cheminement de carrière) qui s'offrent aux personnes vieillissantes dans un modèle d'organisation du travail post-fordiste. Sur ce même thème, Charlotte Nusberg reprend certaines des pratiques d'extension de la vie professionnelle qu'elle avait présentées dans son article paru en 1986 dans le fameux numéro spécial de la revue Ageing International consacré aux travailleurs vieillissants ${ }^{3}$. Enfin, Hélène David et Alain Pilon rapportent les résultats d'une enquête sur les pratiques de gestion en matière de vieillissement dans cinq entreprises manufacturières québécoises. Il s'agit selon nous de la seule étude du genre effectuée au Québec.

La situation, souvent fort précaire, des personnes âgées sur le marché du travail est également analysée. Alan Walker montre les nombreuses difficultés que les travailleurs âgés éprouvent pour retrouver du travail après un licenciement. Retirés du marché du travail, ils sont alors victimes d'un processus d'exclusion sociale qui est encouragé par certaines politiques publiques et les pousse à l'insécurité économique. Zsuzsa Széman illustre cette dernière perspective à partir du cas hongrois où le travail post-retraite devient une nécessité pour de nombreuses personnes âgées qui voient fondre leurs revenus réels face à l'inflation.

Deux derniers thèmes sur l'action collective en faveur des travailleurs à la retraite et sur les femmes et le vieillissement complètent ce tour d'horizon du vieillissement au travail. Les objectifs de ce colloque étaient ambitieux mais ils ont été atteints, les principales dimensions du vieillissement ayant été abordées et fort judicieusement analysées. Notons, toutefois, l'absence de débats autour de questions importantes comme celle des nouvelles technologies qui rendent les conditions de travail moins pénibles et encourageraient l'extension de la vie professionnelle ou celle de l'enrichissement et de la participation qui accroissent la mobilisation et freineraient ainsi le processus d'obsolescence. Notons également une emphase certaine sur le travail de production et les métiers à forte pénibilité qui laisse de côté le travail cadre ou professionnel où la

2 B. CASEY, «Retraite anticipée: les problèmes de la substitution des systèmes et du transfert des coûts et leurs conséquences sur le mécanisme des retraites», Revue internationale de sécurité sociale, $\mathrm{n}^{\circ} 4,1987, \mathrm{p}$. 371-394.

3 C. Nusberg, «Measures that Prolong Work Life», Ageing International, vol. 13, $n^{\circ} 4,1986$, p. $7-10,12-15,17-25$. 
problématique du vieillissement au travail peut apparaître différente. Même si l'approche scientifique et analytique, représentée par les nombreux chercheurs présents, domine largement, la perspective syndicale, centrée sur la solidarité, la défense des droits des travailleurs et le recours à l'État-providence est bien mise en valeur; par contre, celle des employeurs pour qui le vieillissement est également un enjeu important (croissance du coût salarial, adaptation à un environnement économique et technologique en rapide évolution, etc.) est moins prise en considération. Néanmoins, ce colloque de l'IRAT représente une contribution majeure à l'étude du vieillissement au travail et les actes, publiés sous la direction d'Hélène David, constituent une source de référence essentielle pour tout chercheur, enseignant, praticien ou syndicaliste concerné par ce sujet.

Gilles GUÉRIN

Université de Montréal

Le travail en mutation. Nouvelles technologies, qualifications et formation dans les emplois du secteur tertiaire au Québec, par Colette Bernier avec la collaboration de Catherine Teiger, Montréal, Éditions Saint-Martin, 1990, 168 p., ISBN 2-89035-171-8

Il s'agit en fait, de trois études «sectorielles» qui s'intitulent:

1) «Le secteur bancaire, polyvalence et spécialisation des emplois.»

2) «Le secteur des entreprises de service public. Formation en entreprise ou en établissement scolaire?»

3) «Le secteur de l'éducation = intégration des systèmes informatiques et spécialisation des tâches.»

À propos du secteur bancaire, l'auteur conclue au développement de la polyvalence des emplois notamment à partir de «l'étude de l'ancien poste de caissière» et d' «une spécialisation de certains postes dans les services d'épargne et de crédit» (p. 45).

L'étude se limite, il importe de le souligner, à certains postes de caissières et de préposés à l'épargne et au crédit. L'échantillon retenu et traité permet-il de conclure quand il s'agit du secteur bancaire à «la polyvalence et à la spécialisation des emplois»: encore que l'auteur nous dise avoir retenu "plusieurs niveaux d'analyse», soit «le secteur de l'entreprise et le poste de travail» (note infrapaginale p. 25).

De l'étude «des entreprises de service public» dont il est dit, sans plus de précision, qu'elles couvrent «quelques grands monopoles dont les services de téléphone, de gaz et d'électricité» (p. 55), ce qui fait beaucoup, se dégage non pas une conclusion mais une question: «Formation en entreprise ou en établissement scolaire?»

Et le lecteur d'apprendre à partir de la page 59 que l'auteur s'est surtout attardé à l'étude du poste «de commis à la facturation».

De l'étude du secteur de l'éducation l'auteur nous dit à la page 87 qu'il comporte trois niveaux distincts d'emplois: emplois de soutien administratif, emplois de soutien technique et emplois professionnels».

Et de préciser à la page 89 , nous citons et soulignons: 